\title{
Validation of the Ability of a 3D Pedometer to Accurately Determine the Number of Steps Taken by Dairy Cows When Housed in Tie-Stalls
}

\author{
Elise Shepley ${ }^{1, *}$, Marianne Berthelot ${ }^{1,2}$ and Elsa Vasseur ${ }^{1}$ \\ 1 McGill University, Department of Animal Science, Ste-Anne-de-Bellevue, QC H9X 3V9, Canada; \\ marianne.berthelot@agrocampus-ouest.fr (M.B.); elsa.vasseur@mcgill.ca (E.V.) \\ 2 Agrocampus Ouest, 35000 Rennes, France \\ * Correspondence: eshepley1@gmail.com; Tel.: +1-514-398-7796 \\ Academic Editor: Courtney L. Daigle \\ Received: 30 May 2017; Accepted: 27 June 2017; Published: 30 June 2017
}

\begin{abstract}
The automation of farm tasks in dairy production has been on the rise, with an increasing focus on technologies that measure aspects of animal welfare; however, such technologies are not often validated for use in tie-stall farms. The objectives of the current study were to (1) determine the ability of the IceTag 3D pedometer to accurately measure step data for cows in tie-stalls, and (2) determine whether the leg on which the pedometer is mounted impacts step data. Twenty randomly selected Holstein dairy cows were equipped with pedometers on each rear leg and recorded for $6 \mathrm{~h}$ over three 2-h periods. Two observers were trained to measure step activity and the total number of steps per minute were measured. Hourly averages for right and left leg data were analyzed separately using a multivariate mixed model to determine the correlation between pedometer and video step data as well as the correlation between left and right leg step data. The analysis of the video versus pedometer data yielded a high overall correlation for both the left $(r=0.93)$ and right $(r=0.95)$ legs. Additionally, there was good correlation between the left and right leg step data $(r=0.80)$. These results indicate that the IceTag 3D pedometers were accurate for calculating step activity in tie-stall housed dairy cows and can be mounted on either leg of a cow. This study confirms that these pedometers could be a useful automated tool in both a research and commercial setting to better address welfare issues in dairy cows housed in tie-stalls.
\end{abstract}

Keywords: automation; dairy cow; pedometer; validation study

\section{Introduction}

In recent years, the adoption of automation within the dairy industry has increased, changing how cows are milked (e.g., robotic milkers), fed (e.g., automatic feeding systems), and monitored (e.g., ear-, collar- or leg-mounted activity monitors). Greater attention is being paid to production animal welfare, increasing the need for more precise, automated methods of measuring welfare. Lying time, assessed by activity monitors such as pedometers, is one of the most commonly used outcome measure of dairy cow welfare [1]. Step activity, also measured by these devices, may have its own application in welfare monitoring, particularly with regard to lameness detection [2]. The application of automated technologies on dairy farms can be dependent on the type of housing used. Tie-stall housing, as a primary example, inhibits movement ability in dairy cows, restricting the physical and behavioral indicators that can be used by farmers to detect early signs of illness or monitor heat. This is particularly true in the implementation of activity monitoring through pedometers. Step activity for various types of pedometer technologies have been previously validated, primarily targeting use in loose-housing [3] and pasture [4]. Felton et al. [5] provides, to the author's knowledge, the only currently available 
insight on the applicability of pedometers in tie-stall housing systems, and their study found that the AfiMilk pedometers, when compared to observation videos of step activity, accurately measured step activity in tie-stall housed cows. The objective of the current study was to validate the accuracy of the IceTag 3D pedometer (IceRobotics, Edinburgh, UK) in measuring step activity in tie-stall housed dairy cows. This study also sought to determine if the leg on which the pedometer is mounted (i.e., either the right or left leg) effects the step data measurement.

\section{Materials and Methods}

Twenty tie-stall housed Holstein dairy cows were randomly selected from the McGill University Macdonald Campus Dairy Complex (Ste-Anne-de-Bellevue, QC, Canada) for use in the study. Cows ranged in parity (mean \pm SD and range: $2.75 \pm 0.99,1-5$ ) and stage of lactation (DIM (days in milk) mean \pm SD and range; $123.6 \pm 115.45,2-432$ ). Prior to the start of the validation process, leg mounted 3D accelerometers (HOBO Pendant G Acceleration Data Logger, Onset Computer Corp., Pocasset, MA, USA), set to record at 1-min intervals, were attached to the rear leg of eight randomly selected cows for a period of seven days to identify three 2-h periods of time where long bouts of standing were common and activity was likely to be high. The three selected 2-h periods were 10:00 to 12:00, 14:30 to 16:30, and 19:00 to 21:00.

Two different cows were recorded daily for three pre-determined 2-h recording periods using two camcorders (JVC GZ-E100BU AVCHD 40X Optical Zoom, Konica Minolta HD Lens 116 mm 1:1.8, Mississauga, ON, Canada; JVC GZ-R10BU AVCHD 60X Dynamic Zoom, Konica Minolta HD Lens $116 \mathrm{~mm}$ 1:1.8, Mississauga, ON, Canada), amounting to $6 \mathrm{~h}$ of video recording per cow (120 $\mathrm{h}$ total). The camcorders were placed in the alley behind the cows and positioned to ensure that the feet remained in sight for the duration of the recording periods. Before the first 2-h recording period, the cows were equipped with two IceTag 3D pedometers, mounted on to the left and right rear legs of the cows. The exact start time, starting on the minute, was recorded prior to each of the three recordings for all 20 cows. Following each recording day, the pedometers were removed and placed on a new cow. Data from the pedometers were extracted weekly.

Video recordings were reviewed by a single observer. The observer reviewed the $6 \mathrm{~h}$ of video recording for each cow, counting the total number of steps observed each minute. The definition of a step was determined by reviewing second by second pedometer data and corresponding video recordings. In a similar study investigating step activity in tie-stall housed cows, a step was defined as when the rear foot lifted completely off the ground and was returned to the ground in any location with or without moving the entire body [5]. In the initial review of the videos, it was found that it was not necessary for the foot to lift off the ground and, as such, an additional component to this definition of a step was added. In addition to when the foot completely leaves the ground, a step was also counted when the foot moves quickly $(<1 \mathrm{~s})$ and at a distance roughly equivalent to two hoof lengths or more in any direction without leaving the ground. The observer was trained to observe for step activity with a secondary observer also trained in the same manner to ensure that the observation process was reliable and repeatable between observers. Inter-observer reliability calculated using a weighted Kappa coefficient as $K_{\mathrm{w}}=0.82$. Intra-observer repeatability was also calculated using a weighted Kappa coefficient as $K_{\mathrm{w}}=0.88$ and 0.86 for observer 1 and 2, respectively.

\section{Statistical Analysis}

The analysis was run on hourly step averages for both the pedometer and observation data. Pedometer step data for the left leg of two cows were excluded due to technological error and, as such, only 18 cows were used in the analysis of left step data. Left and right leg pedometer data were analyzed separately using a multivariate mixed model with the number of steps as a dependent variable and technology and time as fixed effects. Data was run in SAS (version 9.4, SAS Institute Inc., Cary, NC, USA) using PROC MIXED to obtain covariance estimates from which correlation between observation and pedometer data could be computed. The same multivariate mixed model was used to 
obtain estimates to calculate the correlation between the left foot and the right foot with step as the dependent variable and leg and time as the fixed effects.

\section{Results and Discussion}

The average number of steps recorded in the 6-h observation period and the corresponding average number of steps recorded by the IceTag 3D pedometer for both the left and right leg can be found in Table 1 . The number of steps recorded by the pedometer was found to be highly correlated with the observed number of steps from the video recordings for both the left leg $(r=0.93)$ and the right leg $(r=0.93)$, indicating the pedometer's accuracy in measuring step activity in tie-stall housed dairy cows. These findings are in line with those of Felton et al. [5], which found a similarly high correlation $(r=0.88)$ between video recordings and the AfiMilk pedometers to accurately measure step activity in tie-stall housed cows. It was expected that the IceTag 3D pedometer would yield similar accuracy, as step activity for the AfiMilk pedometer had been previously validated against the IceTag $3 \mathrm{D}$ pedometer in a free-stall housing system and was found to have similar outputs $(r=0.82 ;[3])$.

Table 1. Mean $\pm \mathrm{SD}$, minimum, maximum, and median of the total number of steps performed by the selected tie-stall housed cows in the 6-h recording period for the left and right leg of both the pedometer output and observation video results.

\begin{tabular}{rccccc}
\hline Leg & Step Measurement & Mean \pm SD & Minimum & Maximum & Median \\
\hline \multirow{2}{*}{ Left } & Pedometer & $196.4 \pm 75.2$ & 51 & 354 & 194 \\
& Observation & $219.1 \pm 74.0$ & 103 & 380 & 179.5 \\
\hline \multirow{2}{*}{ Right } & Pedometer & $221.5 \pm 89.2$ & 63 & 383 & 228.5 \\
& Observation & $239.3 \pm 94.3$ & 84 & 436 & 238 \\
\hline
\end{tabular}

The number of steps recorded by the pedometer on the left leg was also well correlated with the number of steps recorded by the pedometer on the right leg $(r=0.80)$. Good correlation between the pedometers on the two legs suggests that the leg on which the pedometer is mounted is not important in ensuring the reliability of the output, necessitating fewer pedometers to accurately measure cow step activity in tie-stall setups. This has important implications, particularly in the monitoring of dairy cow activity both in a research setting and on commercial farms. The use of a single pedometer requires less monetary investment and therefore increases the accessibility of the technology for end-users.

Step activity, measured by the pedometer, may have the potential to be a means of early detection of lameness in dairy cows. Lameness is one of the leading causes of involuntary culling of dairy cows [6] and, as such, automated methods for the early detection of lameness would have considerable benefits to both the cow and the producer. Lying time, an additional measure recorded by most pedometers, is a commonly used indicator of cow welfare, with health issues such as lameness leading to alterations in the amount of time cow spends lying down [1]. In tie-stalls, however, an epidemiological study of 100 tie-stall herds found no relationship between lying measures (total lying time, bout frequency, or mean bout duration) and prevalence of lameness [7]. As noted in Vasseur [1], a wide range of individual variation coupled with additional environmental and physiological factors influence the ability of lying time, on its own, to be entirely reliable for detecting lameness. Similarly, step activity has been linked to lameness, as step activity was found to be significantly decreased in lame cows compared to non-lame cows $(p<0.001)$ in free-stall systems [2]. As with lying time, a large variation between individuals has been found to be a potential issue with the use of step activity as an indicator of lameness [8]. Coupling both aspects of the pedometer technology may have the potential to increase the ability of pedometers to detect lameness in tie-stall housed cows; however, further research is necessary to confirm this assertion.

This study found that IceTag 3D pedometers are indeed accurate in recording step activity in tie-stall housed dairy cows. Furthermore, the results from the left and right leg data yielded similar 
results, allowing for the pedometer to be attached to either rear leg without compromising the step data obtained. Ensuring the validity of the pedometer's application in tie-stall systems opens this technology's use up to new possibilities in future research, particularly in lameness detection for tie-stall housed dairy cows.

Acknowledgments: The authors would like to acknowledge the support of our funding partners Natural Sciences and Engineering Research Council of Canada, Novalait, Dairy Farmers of Canada, and Valacta through Vasseur's Industrial Research Chair. We would also like to extend our thanks to the staff of McGill's Macdonald Campus Dairy.

Author Contributions: Elise Shepley designed the project, oversaw the data collection, supervised the statistical analysis, and contributed to the writing of the manuscript. Marianne Berthelot assisted with the project design, data collections, analyzed the observations videos, and contributed to the writing of the manuscript. Elsa Vasseur oversaw the project, supervised Elise Shepley and Marianne Berthelot through the project design, data collection, and statistical analysis as well as contributed in the writing of the paper.

Conflicts of Interest: The authors declare no conflict of interest.

\section{References}

1. Vasseur, E. Animal behavior and well-being symposium: Optimizing outcome measures of welfare in dairy cattle assessment. J. Anim. Sci. 2017, 95, 1365-1371. [CrossRef] [PubMed]

2. O'Callaghan, K.A.; Cripps, P.J.; Downham, D.Y.; Murray, R.D. Subjective and objective assessment of pain and discomfort due to lameness in dairy cattle. Anim. Welf. 2003, 12, 605-610.

3. Higginson, J.H.; Millman, S.T.; Leslie, K.E.; Kelton, D.F. Validation of a New Pedometry System for Use in Behavioral Research and Lameness Detection in Dairy Cattle. In Proceedings of the First North American Conference on Precision Dairy Management, Toronto, ON, Canada, 2-5 March 2010; pp. 132-133.

4. Elischer, M.F.; Arceo, M.E.; Karcher, E.L.; Siegford, J.M. Validating the accuracy of activity and rumination monitor data from dairy cows housed in a pasture-based automatic milking system. J. Dairy Sci. 2013, 96, 6412-6422. [CrossRef] [PubMed]

5. Felton, C.A.; Colazo, M.G.; Ponce-Barajas, P.; Bench, C.J.; Ambrose, D.J. Dairy cows continuously-housed in tie-stalls failed to manifest activity changes during estrus. Can. J. Anim. Sci. 2012, 92, 189-196. [CrossRef]

6. Langford, F.M.; Stott, A.W. Culled early or culled late: Economic decisions and risks to welfare in dairy cows. Anim. Welf. 2012, 2, 41-55. [CrossRef]

7. Charlton, G.L.; Bouffard, V.; Gibbons, J.; Vasseur, E.; Haley, D.B.; Pellerin, D.; Rushen, J.; de Passillé, A.M. Can automated measures of lying time help assess lameness and leg lesions on tie-stall dairy farms? Appl. Anim. Behav. Sci. 2016, 175, 14-22. [CrossRef]

8. Mazrier, H.; Tal, S.; Aizinbud, E.; Bargai, U. A field investigation of the use of the pedometer for the early detection of lameness in cattle. Can. Vet. J. 2006, 47, 883-886. [PubMed]

(C) 2017 by the authors. Licensee MDPI, Basel, Switzerland. This article is an open access article distributed under the terms and conditions of the Creative Commons Attribution (CC BY) license (http://creativecommons.org/licenses/by/4.0/). 